\title{
Breaking the periodic arrangement of atoms for the enhanced electrochemical reduction of nitrogen and water oxidation
}

\author{
Shilong Jiao ${ }^{1,2,3 \dagger}$, Xianwei $\mathrm{Fu}^{4 \dagger}$, Shuangchen Ruan ${ }^{1}$, Yu-Jia Zeng ${ }^{1 *}$ and Hongwen Huang ${ }^{2,5^{*}}$
}

\begin{abstract}
The development of cost-effective and highperformance electrocatalysts has been increasingly studied to mitigate upcoming energy and environmental challenges. Amorphization and heterointerface engineering have played significant roles in the rational design of electrocatalysts and modulation of their electrocatalytic activities. However, the synergistic effect between amorphization and heterointerfaces has been scarcely reported. As a proof-of-concept attempt, we develop amorphous FeMo (a-FeMo) electrocatalysts with an abundance of heterointerfaces that are composed of amorphous components and evaluate their electrocatalytic performances toward the nitrogen reduction reaction and oxygen evolution reaction (OER). Benefitting from the synergistic effect between the amorphous nature of the a-FeMo electrocatalysts, which offer a high density of active sites, and significant electron redistribution at the heterointerfaces, the electrocatalysts exhibit a high Faradaic efficiency of $29.15 \%$, an elevated yield rate of $71.78 \mu_{\mathrm{NH}_{3}} \mathrm{mg}_{\text {cat. }}{ }^{-1} \mathrm{~h}^{-1}$ with long-term stability at a potential of $-0.1 \mathrm{~V} v$ s. reversible hydrogen electrode and excellent electrocatalytic activity toward the OER. This study provides a promising and effective method for the rational design of low-cost heterogeneous catalysts with desirable efficiency, selectivity, and stability.
\end{abstract}

Keywords: amorphism and heterointerface, nitrogen reduction reaction, OER, electrocatalyst design, electrocatalytic conversion

\section{INTRODUCTION}

The world currently relies heavily on fossil fuels for energy production and chemical industries, such as the ammonia industry, which consumes approximately $1 \%-2 \%$ of anthropogenic energy, while also producing $\sim 400 \mathrm{Mt} \mathrm{CO}_{2}$ and causing serious environmental impacts $[1,2]$. To mitigate the energy crisis and potential ecological catastrophe, the search for alternative energy resources and economic storage and conversion systems has grown to be one of the focuses of scientific society [3-5]. As an essential part of sustainable energy systems, electrochemical energy conversion devices enable the synthesis of value-added chemicals, including but not limited to hydrogen, hydrocarbons, oxygenates, and ammonia, from the abundant feedstock of $\mathrm{H}_{2} \mathrm{O}, \mathrm{CO}_{2}$, and $\mathrm{N}_{2}$, thereby offering a promising strategy for the sustainable development of the world [6-10]. The principal component of electrochemical devices is electrocatalyst that enable energy conversion from one to another, and whose performance is significantly influenced by its chemical composition, size, and morphology along with the type of electrolyte $[11,12]$.

Aiming for the preparation of electrocatalysts with high Faradaic efficiency, long-term stability, and sufficient yield rate, various approaches that aim to maximize the density of active sites, enhance the conductivity and improve the intrinsic activity of the active sites have been applied in the preparation of electrocatalysts [13-17]. Among them, defect and heterointerface engineering have been increasingly studied due to their feasible production, apparent influence on the electrocatalytic performances of electrocatalysts, and low cost; additionally, electrocatalyst engineered in this way has demonstrated advantages in the hydrogen evolution reaction (HER) [18-20], oxygen evolution reaction (OER) [21-26], $\mathrm{CO}_{2}$ reduction reaction (CRR) [27-29], oxygen reduction reaction (ORR) [30-32], and nitrogen reduction reaction (NRR) [33-35]. Benefitting from the higher density of active sites due to the short-range ordering and relatively crumbly structure of these electrocatalysts, amorphization has arisen as an efficient way to modulate them [36-40]. On the other hand, interfaces including crystal boundaries that can stabilize active surface catalytic sites and enable their synergistic effects have also been proven important in tailoring the electrocatalytic properties of electrocatalysts [25,27,29]. Despite the intensive investigation of amorphization and interfaces, their simultaneous combination has rarely been reported, which may trigger interesting electrocatalyst properties.

Herein, as a proof-of-concept attempt, we report the synthesis of amorphous FeMo (a-FeMo) electrocatalysts with an abundance of interfaces and an optimized Fe/Mo ratio. We evaluate their electrocatalytic performances with regard to the NRR and OER. Owing to the synergistic effects of the amorphous nature of the a-FeMo electrocatalysts with a high density of active sites and significant electron redistribution at the heterointerfaces, the electrocatalysts exhibit a high Faradaic efficiency of $29.15 \%$,

\footnotetext{
${ }^{1}$ College of Physics and Optoelectronic Engineering, Shenzhen University, Shenzhen 518060, China

${ }^{2}$ College of Materials Science and Engineering, Hunan University, Changsha 410082, China

${ }^{3}$ Key Lab for Special Functional Materials of Ministry of Education, Collaborative Innovation Center of Nano Functional Materials and Applications, Henan University, Kaifeng 475004, China

${ }^{4}$ National \& Local Joint Engineering Research Center for Applied Technology of Hybrid Nanomaterials, Henan University, Kaifeng 475004, China

${ }^{5}$ Hefei National Laboratory for Physical Sciences at the Microscale, University of Science and Technology of China, Hefei 230026, China

$\dagger$ These authors contributed equally to this work.

* Corresponding authors (emails: yjzeng@szu.edu.cn (Zeng YJ); huanghw@hnu.edu.cn (Huang H))
} 
an elevated yield rate of $71.78 \mu \mathrm{g}_{\mathrm{NH}_{3}} \mathrm{mg}_{\text {cat. }}{ }^{-1} \mathrm{~h}^{-1}$ with long-term stability at a potential of $-0.1 \mathrm{~V} v s$. reversible hydrogen electrode (RHE) and excellent electrocatalytic performance toward the OER. On the basis of the experimental results, we demonstrate the important role that the synergy between the atomic disorder and heterointerfaces plays in activating and enhancing the catalytic performance of electrocatalysts.

\section{EXPERIMENTAL SECTION}

Synthesis of Fe-Mo electrocatalysts with different Fe/Mo ratios The Fe-Mo electrocatalysts were prepared via a simple hydrothermal method. In a typical process, $10 \mathrm{~mL}$ of ultrapure water containing $\mathrm{NaMoO}_{4} \cdot 2 \mathrm{H}_{2} \mathrm{O}(1.96 \mathrm{mmol}, 164 \mathrm{mg}), \mathrm{Fe}\left(\mathrm{NO}_{3}\right)_{3} \cdot 9 \mathrm{H}_{2} \mathrm{O}$ $(0.28 \mathrm{mmol}, 110 \mathrm{mg})$, urea $(0.25 \mathrm{mmol}, 150 \mathrm{mg})$, and $\mathrm{NH}_{4} \mathrm{~F}$ $(1 \mathrm{mmol}, 37.5 \mathrm{mg})$ in a $30.0-\mathrm{mL}$ vial was treated at $50^{\circ} \mathrm{C}$ for $30 \mathrm{~min}$ in an oil bath with continuous stirring until the reactants completely dissolved. Then, the temperature was quickly increased to $120^{\circ} \mathrm{C}$ and maintained for $12 \mathrm{~h}$. The obtained products were washed several times with ethanol and water after being cooled to room temperature. Finally, they were dried at $60^{\circ} \mathrm{C}$ for $12 \mathrm{~h}$ for further characterization. Fe-Mo electrocatalysts with different $\mathrm{Fe} / \mathrm{Mo}$ ratios were synthesized in a similar method by changing the amounts of $\mathrm{NaMoO}_{4} \cdot 2 \mathrm{H}_{2} \mathrm{O}$ and $\mathrm{Fe}\left(\mathrm{NO}_{3}\right)_{3} \cdot 9 \mathrm{H}_{2} \mathrm{O}$. For simplicity, the Fe-Mo electrocatalysts with different Fe/Mo ratios are denoted as a-FeMo- $x$, where $x$ is the Fe/Mo ratio in moles.

\section{Synthesis of the crystalline FeMo-1/7 electrocatalyst}

The crystalline FeMo-1/7 (c-FeMo-1/7) electrocatalyst was prepared via a simple annealing process. Typically, $50 \mathrm{mg}$ of the aFeMo-1/7 electrocatalyst was placed into a tube furnace and heated at $285^{\circ} \mathrm{C}$ for $4 \mathrm{~h}$ in nitrogen with a ramp speed of $5^{\circ} \mathrm{C} \mathrm{min}^{-1}$. After being naturally cooled to room temperature, c-FeMo-1/7 was obtained and ready for further characterization.

\section{Synthesis of the $\mathrm{Fe}_{3} \mathrm{O}_{4}$ and $\mathrm{MoO}_{3}$ electrocatalysts}

For the synthesis of $\mathrm{Fe}_{3} \mathrm{O}_{4}, 20 \mathrm{~mL}$ of ultrapure water containing $\mathrm{FeCl}_{2} \cdot 4 \mathrm{H}_{2} \mathrm{O}$ (130.5 mg), $\mathrm{FeCl}_{3} \cdot 6 \mathrm{H}_{2} \mathrm{O}(269 \mathrm{mg})$, and $\mathrm{NaOH}(8 \mathrm{mg})$ was first stirred for $30 \mathrm{~min}$ and then transferred to a $40-\mathrm{mL}$ Teflon tube and sealed into an autoclave, which was heated to $160^{\circ} \mathrm{C}$ and maintained for $12 \mathrm{~h}$. The obtained products were washed several times with ethanol and water after being cooled to room temperature. Finally, they were dried at $60^{\circ} \mathrm{C}$ for $12 \mathrm{~h}$ before further characterization.

For the synthesis of $\mathrm{MoO}_{3}, 2 \mathrm{~mL}$ of water containing $486 \mathrm{mg}$ of $\left(\mathrm{NH}_{4}\right)_{6} \mathrm{Mo}_{7} \mathrm{O}_{24} \cdot 4 \mathrm{H}_{2} \mathrm{O}$ was stirred until the solute was fully dissolved, after which concentrated $\mathrm{HNO}_{3}(0.6 \mathrm{~mL})$ was dropwise added and further stirred for another $30 \mathrm{~min}$. Then, the solution was transferred to a $20-\mathrm{mL}$ Teflon tube and sealed into the autoclave, which was heated to $90^{\circ} \mathrm{C}$ and maintained for $3 \mathrm{~h}$. The obtained products were washed several times with ethanol and water after being cooled to room temperature. Finally, they were dried at $60^{\circ} \mathrm{C}$ for $12 \mathrm{~h}$ for further characterization.

\section{Characterization methods}

High-resolution high-angle annular dark-field scanning transmission electron microscopy (HAADF-STEM) and tomography images were obtained on a Cs-corrected FEI Titan $80 / 300 \mathrm{kV}$ TEM/STEM microscope. Powder X-ray diffraction (XRD) patterns were recorded using an X-ray diffractometer (SmartLab
(3), Rigaku) operated at $3 \mathrm{~kW}$. X-ray photoelectron spectroscopy (XPS) analyses were performed using a Kratos Ultra DLD spectrometer equipped with a monochromatic $\mathrm{Al} \mathrm{K}$ radiation source. The binding-energy scales were calibrated using the $\mathrm{C} 1 \mathrm{~s}$ peak at $284.6 \mathrm{eV}$, which was from $\mathrm{C}$ contamination. The morphologies of the samples were examined with TEMc (JEOL H-7000).

\section{Electrochemical NRR performance test}

The electrochemical NRR performance tests were carried out in a standard three-electrode system with an electrochemical workstation (CHI660E). Typically, $3.6 \mathrm{mg}$ of the as-obtained electrocatalysts and $1.0 \mathrm{mg}$ carbon black were dispersed in $0.4 \mathrm{~mL}$ ethanol-isopropyl alcohol solution with a volume ratio of $1: 3$. Then, $24 \mu \mathrm{L}$ of Nafion ( $5 \mathrm{wt} \%$ ) was added, and the solution was further sonicated for $60 \mathrm{~min}$. Next, $10 \mu \mathrm{L}$ of the homogeneous dispersion was drop-cast onto a glassy carbon electrode with a diameter of $5.0 \mathrm{~mm}$. The prepared electrodes were dried at room temperature for further tests. The mass loading of the electrocatalysts was determined to be $\sim 0.43 \mathrm{mg} \mathrm{cm}^{-2}$.

The NRR was performed using a two-compartment H-type cell that was separated by a pretreated Nafion 117 membrane. Pt wire and $\mathrm{Ag} / \mathrm{AgCl}$ electrodes were used as the counter electrode and the reference electrode, respectively. All potentials were calibrated to the RHE reference scale by $E(v s$. RHE $)=E(v s$. Ag/ $\mathrm{AgCl})+0.21+0.0593 \times \mathrm{pH}$. The NRR tests were conducted in an $\mathrm{N}_{2}$-saturated $0.1 \mathrm{~mol} \mathrm{~L}^{-1} \mathrm{KOH}$ solution at room temperature under atmospheric pressure. Before the NRR test, $\mathrm{N}_{2}$ was purged into the electrolyte for at least $30 \mathrm{~min}$ to remove the residual air, and controlled potential electrocatalysis was performed at different potentials for $2 \mathrm{~h}$.

\section{Determination of $\mathrm{NH}_{3}$}

The concentration of the produced $\mathrm{NH}_{3}$ was determined by the indophenol blue method. First, $5 \mathrm{~mL}$ of the solution was removed from the reaction vessel. Then, $2 \mathrm{~mL}$ of $\mathrm{NaOH}$ ( $1 \mathrm{~mol} \mathrm{~L}^{-1}$ with $5 \mathrm{wt} \%$ salicylic acid and $5 \mathrm{wt} \%$ sodium citrate), $1 \mathrm{~mL}$ of $\mathrm{NaClO}\left(0.05 \mathrm{~mol} \mathrm{~L}^{-1}\right)$, and $0.2 \mathrm{~mL}$ of an aqueous solution of $1 \mathrm{wt} \%$ sodium nitroferricyanide were added to the solution. After $2 \mathrm{~h}$ at room temperature, the absorption spectrum was measured using a UV-vis spectrophotometer at a wavelength of $655 \mathrm{~nm}$.

\section{Determination of $\mathrm{N}_{2} \mathrm{H}_{4}$}

The $\mathrm{N}_{2} \mathrm{H}_{4}$ produced during the electrocatalysis process was estimated by the Watt and Chrisp method. A mixture of para(dimethylamino) benzaldehyde $(5.99 \mathrm{~g}), \mathrm{HCl}$ (concentrated, $30 \mathrm{~mL})$, and ethanol $(300 \mathrm{~mL})$ was used as the color reagent. Briefly, $2 \mathrm{~mL}$ of the electrolyte after electrolysis was mixed with $2 \mathrm{~mL}$ of the color reactor. The absorbance of the resulting solution was measured at $455 \mathrm{~nm}$. The absolute calibration of this method was achieved using hydrazine monohydrate solutions of known concentrations as standards.

\section{Calculation of the yield rate and Faradaic efficiency}

The Faradaic efficiency (FE) of the products was calculated using the following equation:

$\mathrm{FE}=c_{\mathrm{NH}_{3}} \times V \times N \times F / Q$,

where $c_{\mathrm{NH}_{3}}$ is the measured $\mathrm{NH}_{3}$ concentration, $V$ is the volume of the electrolyte, $N$ is the number of electrons transferred for 
product formation, $F$ is the Faraday constant $\left(96,485 \mathrm{C} \mathrm{mol}^{-1}\right)$, and $Q$ is the quantity of electric charge integrated by the currenttime $(i-t)$ curve.

\section{RESULTS AND DISCUSSION}

\section{Synthesis and structural characterization}

For the synthesis of a-FeMo catalysts with different $\mathrm{Fe} / \mathrm{Mo}$ ratios, $\mathrm{Fe}\left(\mathrm{NO}_{3}\right)_{3} \cdot 9 \mathrm{H}_{2} \mathrm{O}$ and $\mathrm{Na}_{2} \mathrm{MoO}_{4} \cdot 2 \mathrm{H}_{2} \mathrm{O}$ with desired ratios were first dissolved in water, and then urea and ammonium fluoride were subsequently added (Fig. S1). After begin heated in an oil bath for a certain time, the light orange precipitates were collected and washed with ultrapure water and ethanol several times and dried under vacuum for further characterization (details in the Supplementary information). The microstructure of the product obtained was analyzed with XRD, TEM, and high-resolution TEM (HRTEM). As indicated by the low-magnification TEM images, the a-FeMo electrocatalyst (a-FeMo-1/7) with a $\mathrm{Fe} / \mathrm{Mo}$ ratio of $1 / 7$ consists of uniformly distributed nanoparticles with a radius of $\sim 50 \mathrm{~nm}$ (Fig. 1a). The other aFeMo electrocatalysts show similar particle sizes even though they are synthesized with different Fe/Mo ratios (Fig. S2). The fringes of the nanoparticles with HRTEM shows the disordered arrangement of the atoms, indicating the amorphous nature of the a-FeMo-1/7 electrocatalyst (Fig. 1b). The corresponding selected area electron diffraction (SAED) pattern shows no obvious lattice arrangement, again confirming the amorphous nature of the a-FeMo-1/7 electrocatalyst (Fig. 1c). The XRD patterns of the a-FeMo electrocatalysts show no diffraction peaks, as indicated in Fig. S3, implying there is an atomic disorder in the a-FeMo electrocatalysts, regardless of the $\mathrm{Fe} / \mathrm{Mo}$ ratio. The detailed structural information of a-FeMo-1/7 was further revealed by HAADF-STEM. As shown in Fig. 1d, lattice fringes with distinct $d$-spacing values can be readily observed, corresponding to the interplanar distance of (311) in $\mathrm{Fe}_{3} \mathrm{O}_{4}$ and (100) in $\mathrm{MoO}_{3}[41,42]$, thus indicating the composition of the aFeMo-1/7 electrocatalyst. More detailed observations with HAADF-STEM indicate the apparent domains of the crystalline zone and the amorphous zone of the a-FeMo-1/7 electrocatalyst, showing a well-ordered local structure for the crystalline zone and a disordered lattice structure with a random atom arrangement in the amorphous zone (Fig. 1e). The interface between the crystalline-amorphous phase boundaries has been proven to be beneficial for electrocatalytic processes by offering numerous adsorption sites for intermediates during electrocatalytic reactions $[23,43]$. The corresponding intensity profile recorded in Fig. 1e also demonstrates the existence of the crystalline-amorphous phase boundary and the irregular arrangement of atoms in the amorphous zone (Fig. 1f). The corresponding elemental mapping images of a-FeMo-1/7 reveal that $\mathrm{Fe}, \mathrm{Mo}$, and $\mathrm{O}$ are uniformly distributed (Fig. 1g). The atomic ratio of $\mathrm{Fe} / \mathrm{Mo}$ is estimated to be $1 / 6.79$ based on energy
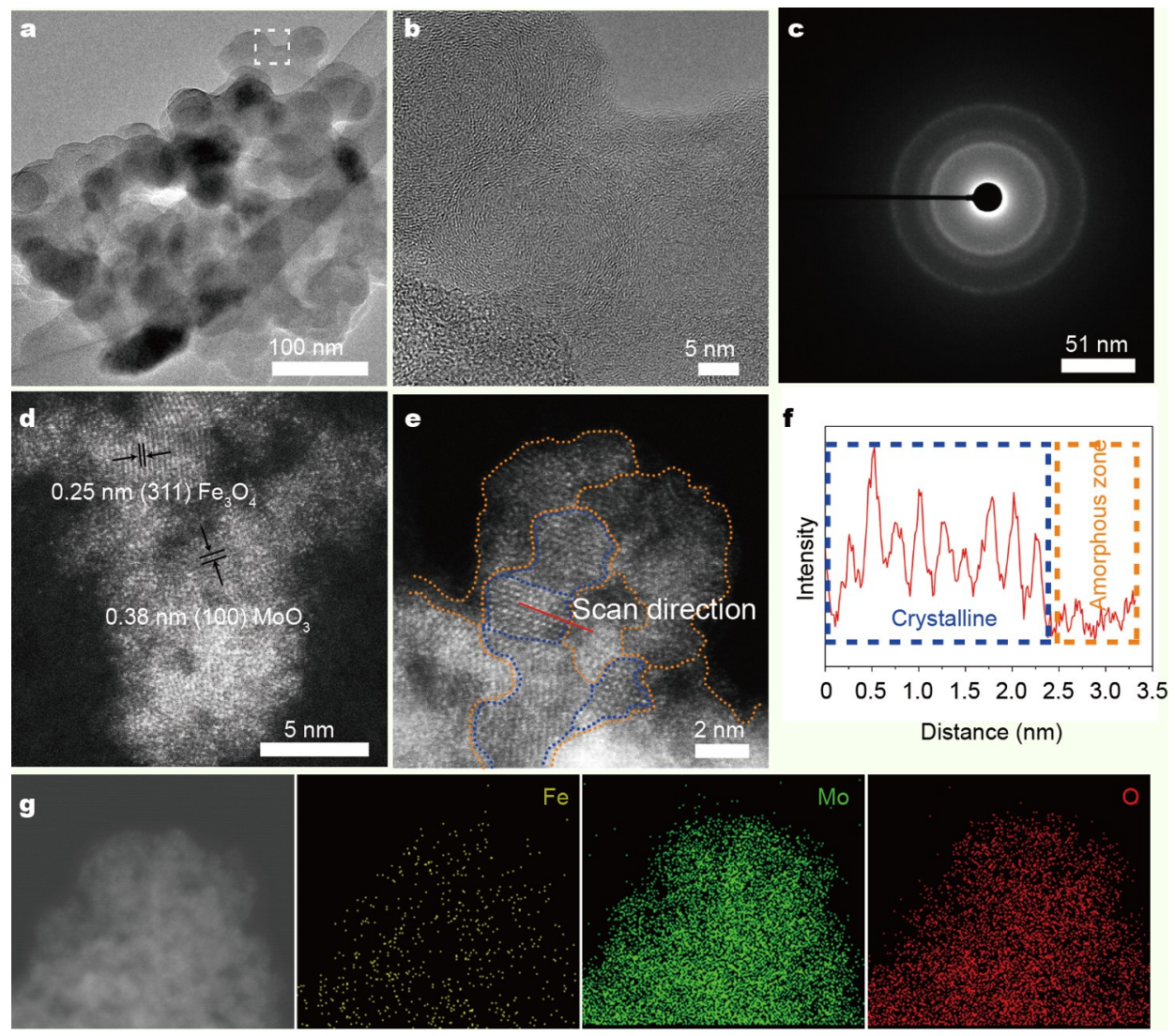

Figure 1 (a) Low-magnification TEM image of the a-FeMo-1/7 electrocatalyst; (b) enlarged HRTEM image of the a-FeMo-1/7 electrocatalyst marked in (a), indicating the amorphous nature of the electrocatalyst; (c) SAED pattern of the a-FeMo-1/7 electrocatalyst; (d) atomic resolution HAADF-STEM image of the a-FeMo-1/7 electrocatalyst with clear lattice fringes; (e) atomic resolution HAADF-STEM image of the a-FeMo-1/7 electrocatalyst, indicating the boundary between the amorphous region and the crystalline region; ( $\mathrm{f}$ ) intensity profile recorded from the line in (e); (g) EDS mapping of the a-FeMo-1/7 electrocatalyst. 
dispersive spectroscopy (EDS), implying the successful preparation of the a-FeMo electrocatalyst with a Fe/Mo ratio of 1/7.

\section{Chemical and electronic configuration}

To probe the amorphism-induced change in the electronic structure of the a-FeMo-1/7 electrocatalyst, XPS and photoluminescence (PL) spectra were collected. First, crystalline $\mathrm{Fe}_{3} \mathrm{O}_{4}$ and $\mathrm{MoO}_{3}$ were prepared via a traditional solvothermal method and used as references. By annealing a-FeMo-1/7 at a high temperature for $4 \mathrm{~h}$, the $\mathrm{c}-\mathrm{FeMo}-1 / 7$ electrocatalyst can be obtained (Fig. S4, with details in the Supplementary information). The XRD patterns of the as-obtained $\mathrm{Fe}_{3} \mathrm{O}_{4}$ and $\mathrm{MoO}_{3}$ indicate the pure phase of these nanomaterials with high crystallinity and no obvious impurities (Fig. S5). As shown in Fig. 2a, the binding energies at $710.4,723.5,712.9,725.4$, and $718.9 \mathrm{eV}$ can be assigned to $\mathrm{Fe}^{2+} 2 \mathrm{p}_{3 / 2}, \mathrm{Fe}^{2+} 2 \mathrm{p}_{1 / 2}, \mathrm{Fe}^{3+} 2 \mathrm{p}_{3 / 2}, \mathrm{Fe}^{3+} 2 \mathrm{p}_{1 / 2}$ and the satellite peak of $\mathrm{Fe} 2 \mathrm{p}$ in pure $\mathrm{Fe}_{3} \mathrm{O}_{4}$, respectively [44-46]. The Fe $2 \mathrm{p}$ peaks in the a-FeMo-1/7 shift positively to higher energies by approximately $0.4 \mathrm{eV}$, indicating the significant alteration of the electron distributions. The a-FeMo electrocatalysts with different $\mathrm{Fe} / \mathrm{Mo}$ ratios demonstrate similar energy shifts, as shown in Fig. S6, validating the fact that the amorphous nature of the electrocatalysts shows a similar influence on their electronic structure. However, the crystalline FeMo electrocatalyst with a $\mathrm{Fe} / \mathrm{Mo}$ ratio of $1 / 7$ shows a negligible energy shift compared with pure $\mathrm{Fe}_{3} \mathrm{O}_{4}$ (Fig. S7), indicating the slight effect of the crystalline interface on the electron distributions. The binding energies at 232.2 and $235.6 \mathrm{eV}$ from the Mo $3 \mathrm{~d}$ spectrum can be assigned to $\mathrm{Mo}^{6+} 3 \mathrm{~d}_{5 / 2}$ and $\mathrm{Mo}^{6+} 3 \mathrm{~d}_{3 / 2}$ in pure $\mathrm{MoO}_{3}$ $[21,47]$ as shown in Fig. 2b. Unsurprisingly, the Mo 3d peak of aFeMo-1/7 shows a significantly negative shift towards lower binding energies, indicating electron transfer from the Fe to Mo atoms. The Mo 3d peaks of other FeMo electrocatalysts with different Fe/Mo ratios show the same trend, again validating the significance of amorphism on the electron distribution of the electrocatalysts (Fig. S6). The Mo 3d spectrum of c-FeMo-1/7 shows little change in the peak positions, implying the limited electronic interaction between crystalline $\mathrm{Fe}_{3} \mathrm{O}_{4}$ and $\mathrm{MoO}_{3}$ (Fig. S8). Different from the Fe $2 \mathrm{p}$ and Mo $3 \mathrm{~d}$ peaks, the $\mathrm{O} 1 \mathrm{~s}$ peak of the FeMo electrocatalysts is located at almost the same position with binding energies of 530.1 and $531.6 \mathrm{eV}$, which can be ascribed to the lattice oxygen and hydroxyl groups (Fig. 2c and Figs S6 and S7) [21,45]. Fig. 2d shows the PL spectra of aFeMo-1/7 and c-FeMo-1/7, which display a strong PL emission peak at $\sim 435 \mathrm{~nm}$ that corresponds to the recombination of holes in the disordered area of the electrocatalyst [46]. The PL spectra of the FeMo electrocatalysts with different Fe/Mo ratios also show obvious PL emission peaks, as indicated in Fig. S9. Combined with the XPS and PL spectra, the strong electronic interaction between $\mathrm{Fe}_{3} \mathrm{O}_{4}$ and $\mathrm{MoO}_{3}$ and the electron accumulation on the Mo sites can be validated, which implies the potential for enhanced electrocatalytic performance.

\section{NRR electrochemical evaluation}

To validate the assumption that the amorphism and interface can serve as an advantage in the electrocatalytic process, the electrochemical activity of a-FeMo-1/7 was evaluated with regard to the NRR and OER. The electrocatalytic activities of the samples were carried out in an $\mathrm{N}_{2}$-saturated $0.1 \mathrm{~mol} \mathrm{~L}^{-1} \mathrm{KOH}$ solution in a typical $\mathrm{H}$-type cell at room temperature and ambient pressure. The produced $\mathrm{NH}_{3}$ was spectrophotometrically determined by the indophenol blue method, and the possible production of $\mathrm{N}_{2} \mathrm{H}_{4}$ was detected by spectrophotometry with dimethylaminobenzaldehyde $[27,38,48]$. The
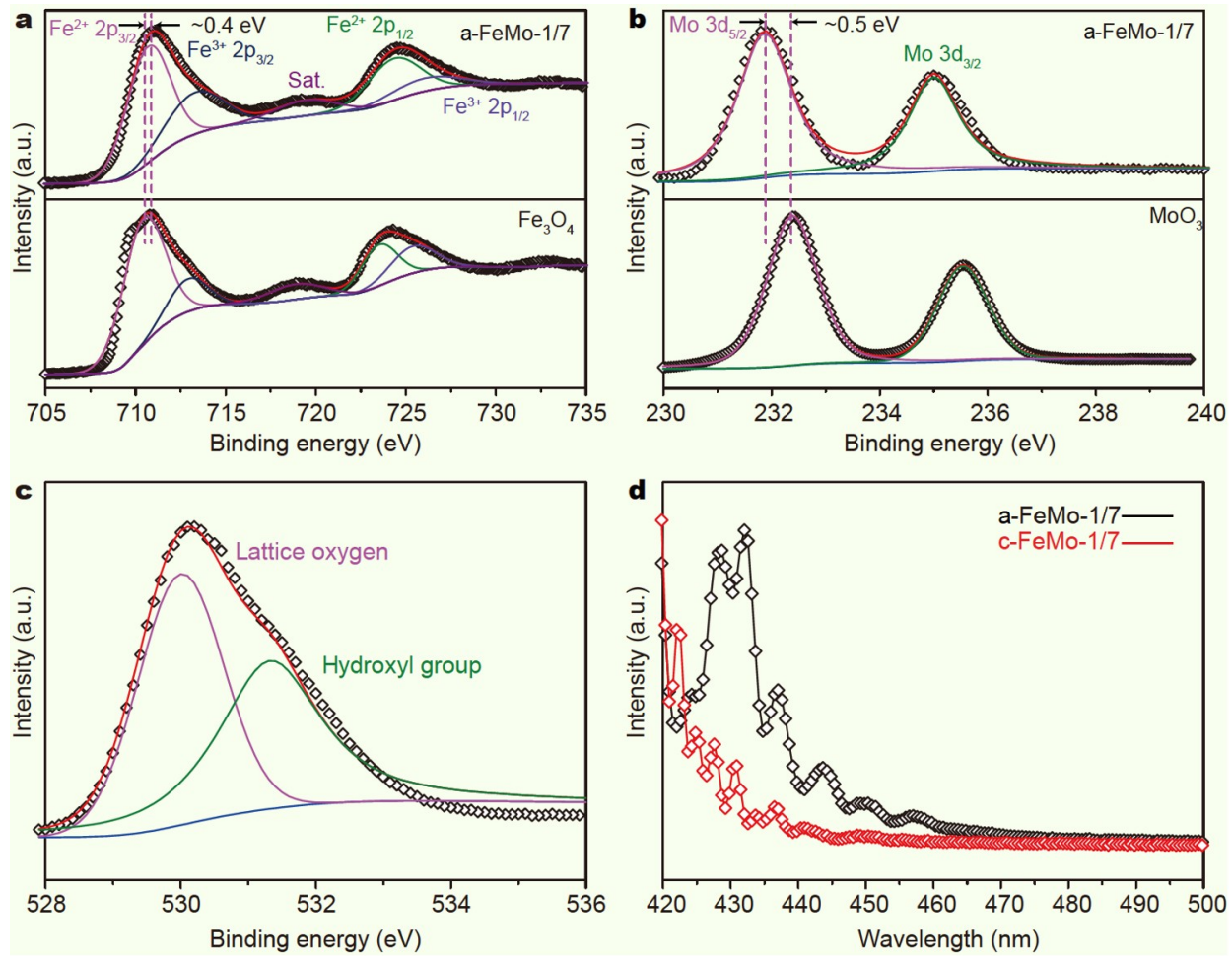

Figure 2 (a) Fe 2p XPS spectra of the a-FeMo-1/7 electrocatalyst and $\mathrm{Fe}_{3} \mathrm{O}_{4}$; (b) Mo $3 \mathrm{~d}$ XPS spectra of the a-FeMo-1/7 electrocatalyst and MoO 3 ; (c) O 1s XPS spectra of the a-FeMo-1/7 electrocatalyst; (d) PL spectra of the a-FeMo-1/7 and c-FeMo-1/7 electrocatalysts. 
corresponding calibration curves are displayed in Figs S10 and S11. All potentials in this work were calibrated to the values versus RHE with no exceptions. The linear sweep voltammetry (LSV) curves of the FeMo electrocatalysts in Ar-saturated and $\mathrm{N}_{2}$-saturated electrolytes demonstrate different current densities, which indicates the obvious reduction of nitrogen (Fig. S12). The a-FeMo-1/7 shows a higher current density than the other a-FeMo electrocatalysts, implying its higher activity toward the NRR. Since the HER can be a major competing reaction with the NRR, a sequence of potentials was applied to achieve the optimum conditions for $\mathrm{NH}_{3}$ production.

As shown in Fig. 3a, the highest average yield rate of $\mathrm{NH}_{3}$ for a-FeMo-1/7 is $71.78 \mu \mathrm{g}_{\mathrm{NH}_{3}} \mathrm{mg}_{\text {cat. }}{ }^{-1} \mathrm{~h}^{-1}$, with a corresponding Faradaic efficiency of $29.15 \%$ (Fig. $3 b$ ) at $-0.1 \mathrm{~V} v s$. RHE; this electrocatalyst outperforms the a-FeMo electrocatalysts with different Fe/Mo ratios (Fig. S13). Thus, a-FeMo-1/7 is one of the best NRR electrocatalysts compared with other reported electrocatalysts (Table S1). To exclude artifacts, such as indoor ambient ammonia contamination and other sources of nitrogen, control experiments were carried out. The UV-vis spectra of the electrolyte after the NRR test in Ar and $\mathrm{N}_{2}$ are shown in Fig. S14. The results indicate that a negligible amount of $\mathrm{NH}_{3}$ is detected, thereby demonstrating that $\mathrm{NH}_{3}$ mainly comes from the electrochemical reduction of $\mathrm{N}_{2}$. As indicated in Fig. 3c, the aFeMo-1/7 further shows excellent durability during long-term electrolysis at different potentials, which is better than the aFeMo electrocatalysts with other Fe/Mo ratios (Fig. S13). Further investigation of the stability is displayed in Fig. S15, with $36 \mathrm{~h}$ of continuous electrolysis at $-0.1 \mathrm{~V}$ vs. RHE. The chronoamperometry curve shows no obvious drop in current density, which confirms its strong stability. Further characterizations reveal a negligible change in the morphology and XPS peaks after the NRR durability test, illustrating its excellent stability (Fig. S16). Both the $\mathrm{NH}_{3}$ yield rate and the $\mathrm{FE}$ values are also well maintained for $12 \mathrm{~h}$ ( 6 cycles) of the continuous electrochemical NRR test (Fig. 3d). To rationalize the excellent performance of the a-FeMo electrocatalyst during the NRR evaluation, the electrochemically active surface area (ECSA), which reveals the presence of active sites in electrocatalysts, was calculated by measuring the double-layer capacitance $\left(C_{\mathrm{dl}}\right)$. The value of $C_{\mathrm{dl}}$ for a-FeMo-1/7 is $1.78 \mathrm{mF} \mathrm{cm}^{-2}$, which is higher than that of the a-FeMo electrocatalysts with other Fe/Mo ratios $\left(1.45 \mathrm{mF} \mathrm{cm}^{-2}\right.$ for a-FeMo-1/15, $1.69 \mathrm{mF} \mathrm{cm}^{-2}$ for a-FeMo-1/3, and $1.51 \mathrm{mF} \mathrm{cm}^{-2}$ for a-FeMo-1/1), as shown in Figs S17 and S18. This result reveals the higher density of active sites for aFeMo-1/7. The largest ECSA value of the a-FeMo-1/7 electrocatalyst indicates the maximum number of active sites, which may originate from the disordered arrangement of atoms [46]. Despite the high density of active sites, the electrical transport properties revealed by electrochemical impedance spectroscopy (EIS) verify that the a-FeMo-1/7 electrocatalyst exhibits lower charge-transfer resistance and higher charge transport properties at different overpotentials (Fig. S19). Taken together, the aFeMo-1/7 electrocatalyst demonstrates the most advanced NRR performance among various compositions, which results from its high density of active sites, better electronic conductivity, and smaller electron transport resistance.

To further address the importance of the synergy between the atomic disorder and heterointerfaces, the NRR activities of pure $\mathrm{Fe}_{3} \mathrm{O}_{4}, \mathrm{MoO}_{3}$, and c-FeMo-1/7 were also investigated and compared with the state-of-the-art a-FeMo-1/7 electrocatalyst. The LSV curves indicate a smaller difference in current density compared with a-FeMo-1/7, which implies lower NRR activity (Fig. S20). As shown in Fig. 4a and Fig. S21, pure $\mathrm{Fe}_{3} \mathrm{O}_{4}$ and
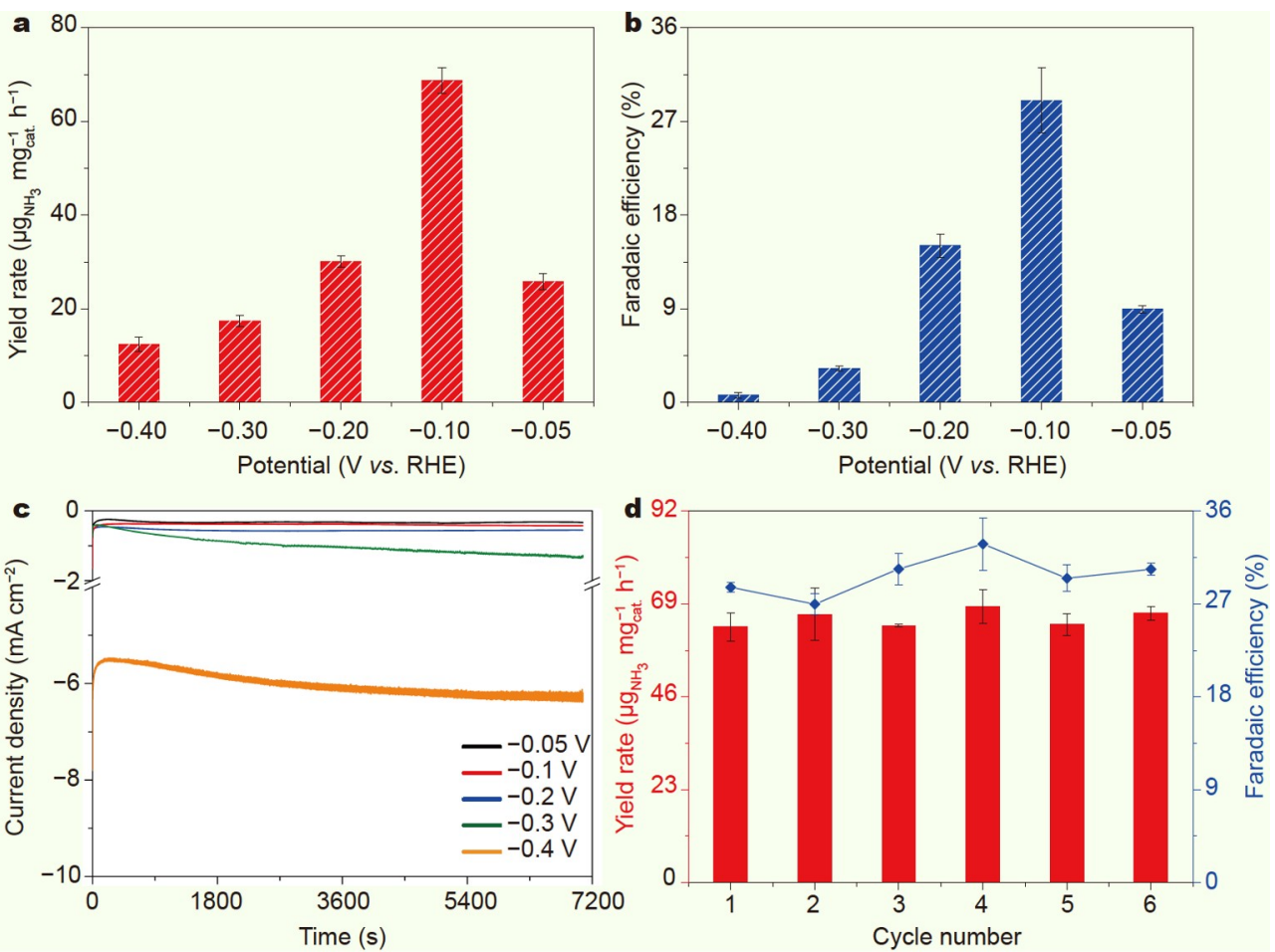

Figure 3 (a) $\mathrm{NH}_{3}$ yield rate of the a-FeMo-1/7 electrocatalyst at different potentials; (b) Faradaic efficiencies of the a-FeMo-1/7 electrocatalyst at different potentials; (c) chronoamperometric curves of the a-FeMo-1/7 electrocatalyst at different applied potentials; (d) recycling test of the a-FeMo-1/7 electrocatalyst at a potential of $-0.1 \mathrm{~V} v s$. RHE. 
$\mathrm{MoO}_{3}$ exhibit inferior NRR activity with yield rates of 14.72 and $9.72 \mu \mathrm{g}_{\mathrm{NH}_{3}} \mathrm{mg}_{\text {cat. }}{ }^{-1} \mathrm{~h}^{-1}$ at potentials of $-0.1 \mathrm{~V} v s$. RHE. These values are much lower than that of a-FeMo-1/7, indicating a weak interaction between nitrogen and these electrocatalysts. Notably, c-FeMo-1/7 only shows a yield rate of $5.77 \mu \mathrm{g}_{\mathrm{NH}_{3}} \mathrm{mg}_{\text {cat. }}{ }^{-1} \mathrm{~h}^{-1}$ at a potential of $-0.1 \mathrm{~V} v s$. RHE, while aFeMo-1/7 shows a much higher yield rate (12.4 times higher), which highlights the much stronger electron interaction between $\mathrm{N}_{2}$ molecules and the surface of a-FeMo-1/7 [43]. As expected, a-FeMo-1/7 delivered the highest Faradaic efficiency at a potential of $-0.1 \mathrm{~V} v s$. RHE (Fig. $4 \mathrm{~b}$ ). The calculated effective density $\left(j_{\mathrm{N}}\right)$ of the a-FeMo-1/7 electrocatalyst is $0.116 \mathrm{~mA} \mathrm{~cm}^{-2}$, which is 9.99 times higher than that of the c-FeMo-1/7 electrocatalyst (Fig. 4c), again demonstrating its superior NRR performance. Surprisingly, although the ECSA of the a-FeMo-1/7 electrocatalyst is still larger than that of c-FeMo-1/7, it is smaller than that of $\mathrm{Fe}_{3} \mathrm{O}_{4}$ and $\mathrm{MoO}_{3}$ (Figs S22 and S23), demonstrating the higher intrinsic activity of the active sites in the a-FeMo-1/7 electrocatalyst. Similarly, the EIS results at different potentials indicate that the a-FeMo-1/7 electrocatalyst exhibits the lowest charge transfer property but inferior electron transport behavior (Fig. S24). As commonly documented, the activation of the $\mathrm{N} \equiv \mathrm{N}$ triple bond lies as the main obstacle for nitrogen reduction due to the high bond energy [27,48]; thus, activation of this bond requires a stronger interaction between $\mathrm{N}_{2}$ and the surface of the electrocatalyst. By constructing a heterointerface between $\mathrm{Fe}_{3} \mathrm{O}_{4}$ and $\mathrm{MoO}_{3}$, significant electron transfer from the Fe sites to the Mo sites is induced; thus, an excellent NRR electrocatalyst with high Faradaic efficiency of $29.15 \%$, an elevated yield rate of $71.78 \mu \mathrm{g}_{\mathrm{NH}_{3}} \mathrm{mg}_{\text {cat. }}{ }^{-1} \mathrm{~h}^{-1}$, and long-term stability can be readily achieved.

\section{OER electrochemical evaluation}

To further validate the advantage of the synergistic effect between the atomic disorder and heterointerfaces in the electrocatalysis process, a-FeMo-1/7, c-FeMo-1/7, $\mathrm{Fe}_{3} \mathrm{O}_{4}$, and $\mathrm{MoO}_{3}$ were also subjected to OER investigations. The electrocatalytic activity of each sample was evaluated using a three-electrode system with a mass loading of $\sim 0.36 \mathrm{mg} \mathrm{cm}^{-2}$ in an alkaline electrolyte. The detailed experimental procedure for the electrochemical measurement can be found in EXPERIMENTAL SECTION. The LSV curves of a-FeMo-1/7, c-FeMo-1/7, $\mathrm{Fe}_{3} \mathrm{O}_{4}$, and $\mathrm{MoO}_{3}$ are displayed in Fig. 5a, which shows that only a $338 \mathrm{mV}$ overpotential $(\eta)$ is required for a-FeMo-1/7 to achieve an anodic current density of $10 \mathrm{~mA} \mathrm{~cm}^{-2}$. Importantly, the water oxidation activity of a-FeMo-1/7 outperforms that of c-FeMo$1 / 7(390 \mathrm{mV})$ and $\mathrm{Fe}_{3} \mathrm{O}_{4}(470 \mathrm{mV})$ under the same conditions. Further analysis demonstrates an enhancement of the mass activity of the a-FeMo-1/7 electrocatalyst in comparison with their counterparts (Fig. S25). The Tafel slopes of the samples are shown in Fig. 5b, indicating that a-FeMo-1/7 can be a potential electrocatalyst for electrolyzers with a smaller Tafel slope (46.7 $\mathrm{mV} \mathrm{dec}^{-1}$ for a-FeMo-1/7, $86.1 \mathrm{mV} \mathrm{dec}^{-1}$ for c-FeMo-1/7, $103.2 \mathrm{mV} \mathrm{dec}^{-1}$ for $\mathrm{Fe}_{3} \mathrm{O}_{4}$, and $129.8 \mathrm{mV} \mathrm{dec}^{-1}$ for $\mathrm{MoO}_{3}$ ), thereby reducing power loss [46]. Besides, long-term stability can also be achieved for the a-FeMo-1/7 electrocatalyst for as long as $30 \mathrm{~h}$ (Fig. S26). The TEM characterization and the XPS patterns further validate its superior stability in both the morphology and composition (Fig. S27). The excellent OER activity of a-FeMo-1/7 once again proves the advantages of atomic disorder and heterointerfaces in the electrocatalytic process, which may be beneficial for further investigations of high-performance catalysts and provide insight for the design of highly
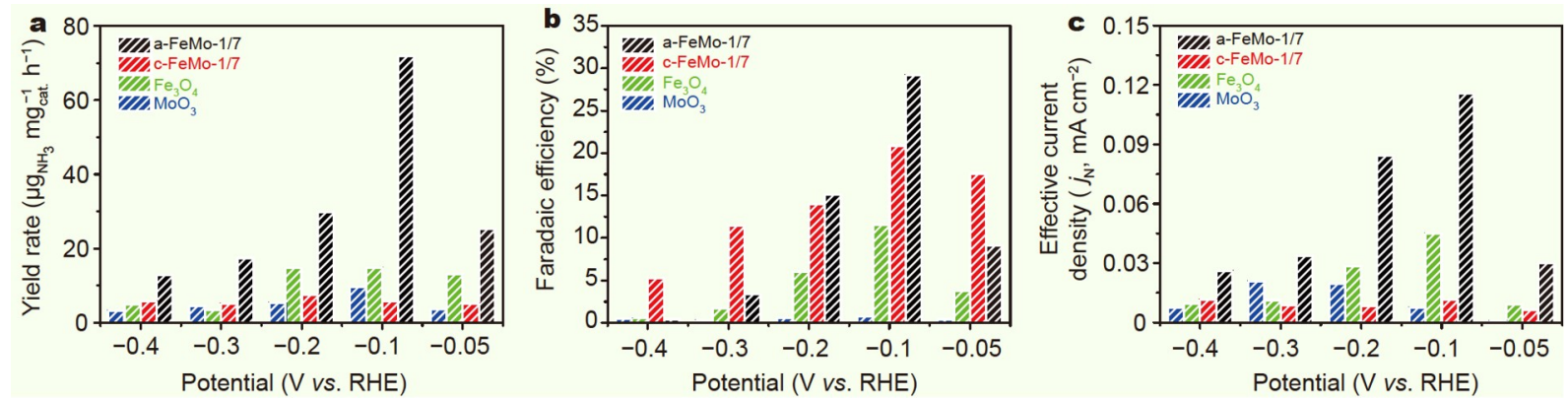

Figure 4 (a) NRR production rate of the a-FeMo-1/7, c-FeMo-1/7, $\mathrm{Fe}_{3} \mathrm{O}_{4}$, and $\mathrm{MoO}_{3}$ electrocatalysts; (b) NRR Faradaic efficiency of the a-FeMo-1/7, cFeMo-1/7, $\mathrm{Fe}_{3} \mathrm{O}_{4}$, and $\mathrm{MoO}_{3}$ electrocatalysts; (c) effective $j_{\mathrm{N}}$ of the a-FeMo-1/7, c-FeMo-1/7, $\mathrm{Fe}_{3} \mathrm{O}_{4}$, and $\mathrm{MoO}_{3}$ electrocatalysts.
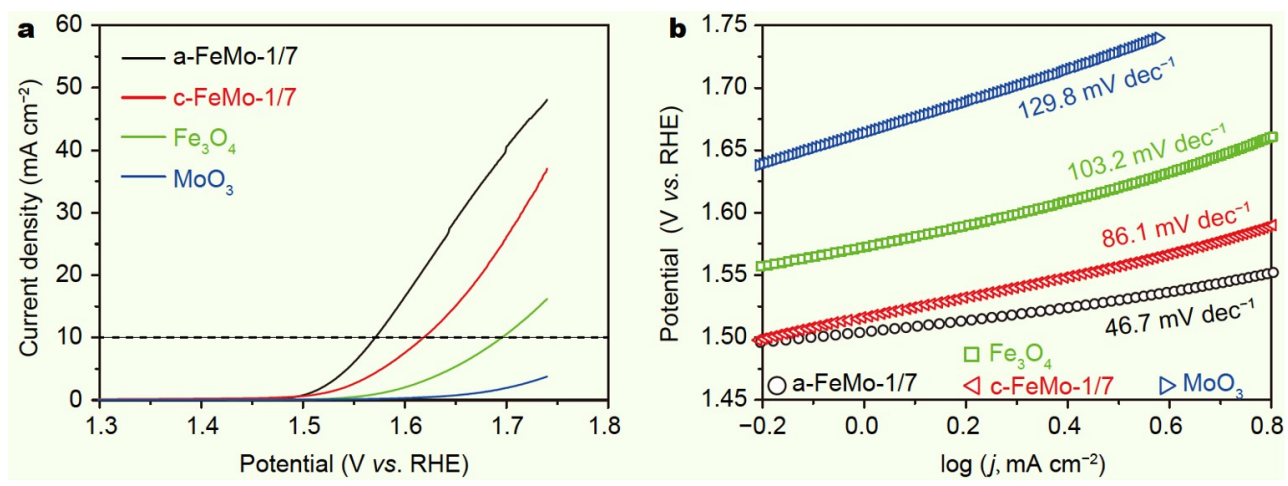

Figure 5 Polarization curves (a) and corresponding Tafel plots (b) of the electrocatalysts during the electrocatalytic OER process. 
efficient electrocatalysts via structural modulation.

\section{CONCLUSIONS}

In summary, we demonstrated that amorphism and heterointerfaces in electrocatalysts could serve as an efficient strategy for the rational design of high-performance electrocatalysts. We report the successful preparation of a-FeMo electrocatalysts with an optimized $\mathrm{Fe} / \mathrm{Mo}$ ratio. The a-FeMo-1/7 electrocatalyst exhibited excellent NRR activity with a high Faradaic efficiency of $29.15 \%$, an elevated yield rate of $71.78 \mu \mathrm{g}_{\mathrm{NH}_{3}} \mathrm{mg}_{\text {cat. }}{ }^{-1} \mathrm{~h}^{-1}$, and long-term stability. These excellent properties originate from the amorphous nature of the electrocatalyst, which increases the density of active sites, and the existence of heterointerfaces that enables obvious electron redistribution between components. Further examination of the OER performance of the studied electrocatalysts again validates the argument. This proof-ofconcept approach not only achieves an active and durable NRR and OER catalyst but also provides a promising and effective alternative method for the design of remarkable heterogeneous catalysts.

\section{Received 7 April 2021; accepted 3 June 2021;} published online 16 August 2021

1 Yan Z, Ji M, Xia J, et al. Recent advanced materials for electrochemical and photoelectrochemical synthesis of ammonia from dinitrogen: One step closer to a sustainable energy future. Adv Energy Mater, 2020, 10: 1902020

2 Meng SL, Li XB, Tung CH, et al. Nitrogenase inspired artificial photosynthetic nitrogen fixation. Chem, 2021, 7: 1431-1450

3 Wang Y, Su H, He Y, et al. Advanced electrocatalysts with single-metalatom active sites. Chem Rev, 2020, 120: 12217-12314

4 Banerjee A, Wang X, Fang C, et al. Interfaces and interphases in allsolid-state batteries with inorganic solid electrolytes. Chem Rev, 2020, 120: 6878-6933

5 MacFarlane DR, Cherepanov PV, Choi J, et al. A roadmap to the ammonia economy. Joule, 2020, 4: 1186-1205

6 Escalera-López D, Lou Z, Rees NV. Benchmarking the activity, stability, and inherent electrochemistry of amorphous molybdenum sulfide for hydrogen production. Adv Energy Mater, 2019, 9: 1802614

7 Herlem G, Alhedabi T, Picaud F. From anodic oxidation of aliphatic aamino acids to polypeptides by quantum electrochemistry approach: Beyond Miller-Urey experiments. J Am Chem Soc, 2019, 141: 1423014238

8 Milton RD, Minteer SD. Nitrogenase bioelectrochemistry for synthesis applications. Acc Chem Res, 2019, 52: 3351-3360

9 Wang N, Zhou R, Li H, et al. New insights into the electrochemistry of carbonyl- and amino-containing polymers for rechargeable zincorganic batteries. ACS Energy Lett, 2021, 6: 1141-1147

10 Qiao $\mathrm{Y}, \mathrm{Yi} J, \mathrm{Wu}$ S, et al. $\mathrm{Li}-\mathrm{CO}_{2}$ electrochemistry: A new strategy for $\mathrm{CO}_{2}$ fixation and energy storage. Joule, 2017, 1: 359-370

11 Jiao S, Fu X, Zhang L, et al. Point-defect-optimized electron distribution for enhanced electrocatalysis: Towards the perfection of the imperfections. Nano Today, 2020, 31: 100833

12 Jiao S, Fu X, Zhang L, et al. The lab-to-fab journey of copper-based electrocatalysts for multi-carbon production: Advances, challenges, and opportunities. Nano Today, 2021, 36: 101028

13 Yang $\mathrm{Q}, \mathrm{Wu} \mathrm{Q}$, Liu $\mathrm{Y}$, et al. Novel Bi-doped amorphous $\mathrm{SnO}_{x}$ nanoshells for efficient electrochemical $\mathrm{CO}_{2}$ reduction into formate at low overpotentials. Adv Mater, 2020, 32: 2002822

14 Chen J, Wang H, Wang Z, et al. Redispersion of Mo-based catalysts and the rational design of super small-sized metallic Mo species. ACS Catal, 2019, 9: 5302-5307

15 Hollevoet L, De Ras M, Roeffaers M, et al. Energy-efficient ammonia production from air and water using electrocatalysts with limited
Faradaic efficiency. ACS Energy Lett, 2020, 5: 1124-1127

16 Han X, Gerke CS, Banerjee S, et al. Strategic design of $\mathrm{MoO}_{2}$ nanoparticles supported by carbon nanowires for enhanced electrocatalytic nitrogen reduction. ACS Energy Lett, 2020, 5: 3237-3243

17 Peng W, Luo M, Xu X, et al. Spontaneous atomic ruthenium doping in $\mathrm{Mo}_{2} \mathrm{CT}_{X}$ MXene defects enhances electrocatalytic activity for the nitrogen reduction reaction. Adv Energy Mater, 2020, 10: 2001364

18 Yadav V, Lowe JS, Shumski AJ, et al. Modulating the structure and hydrogen evolution reactivity of metal chalcogenide complexes through ligand exchange onto colloidal Au nanoparticles. ACS Catal, 2020, 10: 13305-13313

19 Yang G, Jiao $\mathrm{Y}$, Yan $\mathrm{H}$, et al. Interfacial engineering of $\mathrm{MoO}_{2}-\mathrm{FeP}$ heterojunction for highly efficient hydrogen evolution coupled with biomass electrooxidation. Adv Mater, 2020, 32: 2000455

20 Zhang W, Huang B, Wang K, et al. $\mathrm{WO}_{x}$-surface decorated PtNi@Pt dendritic nanowires as efficient $\mathrm{pH}$-universal hydrogen evolution electrocatalysts. Adv Energy Mater, 2021, 11: 2003192

21 Li X, Wang Y, Wang J, et al. Sequential electrodeposition of bifunctional catalytically active structures in $\mathrm{MoO}_{3} / \mathrm{Ni}-\mathrm{NiO}$ composite electrocatalysts for selective hydrogen and oxygen evolution. Adv Mater, 2020, 32: 2003414

22 Zhang L, Lu C, Ye F, et al. Vacancies boosting strategy enabling enhanced oxygen evolution activity in a library of novel amorphous selenite electrocatalysts. Appl Catal B-Environ, 2021, 284: 119758

$23 \mathrm{Hu} \mathrm{Y}$, Luo G, Wang L, et al. Single Ru atoms stabilized by hybrid amorphous/crystalline FeCoNi layered double hydroxide for ultraefficient oxygen evolution. Adv Energy Mater, 2021, 11: 2002816

24 Wang J, Kim SJ, Liu J, et al. Redirecting dynamic surface restructuring of a layered transition metal oxide catalyst for superior water oxidation. Nat Catal, 2021, 4: 212-222

25 Cui X, Ren P, Ma C, et al. Robust interface Ru centers for highperformance acidic oxygen evolution. Adv Mater, 2020, 32: 1908126

26 Zhou Y, López N. The role of $\mathrm{Fe}$ species on $\mathrm{NiOOH}$ in oxygen evolution reactions. ACS Catal, 2020, 10: 6254-6261

27 Liu C, Wu Y, Sun $\mathrm{K}$, et al. Constructing $\mathrm{FeN}_{4} /$ graphitic nitrogen atomic interface for high-efficiency electrochemical $\mathrm{CO}_{2}$ reduction over a broad potential window. Chem, 2021, 7: 1297-1307

28 Ma M, Clark EL, Therkildsen KT, et al. Insights into the carbon balance for $\mathrm{CO}_{2}$ electroreduction on $\mathrm{Cu}$ using gas diffusion electrode reactor designs. Energy Environ Sci, 2020, 13: 977-985

29 Vasileff A, Xu C, Jiao Y, et al. Surface and interface engineering in copper-based bimetallic materials for selective $\mathrm{CO}_{2}$ electroreduction. Chem, 2018, 4: 1809-1831

30 Zhao Z, Hossain MD, Xu C, et al. Tailoring a three-phase microenvironment for high-performance oxygen reduction reaction in proton exchange membrane fuel cells. Matter, 2020, 3: 1774-1790

31 Dong C, Liu ZW, Liu JY, et al. Modest oxygen-defective amorphous manganese-based nanoparticle mullite with superior overall electrocatalytic performance for oxygen reduction reaction. Small, 2017, 13. 1603903

32 Rossi K, Asara GG, Baletto F. Structural screening and design of platinum nanosamples for oxygen reduction. ACS Catal, 2020, 10: 39113920

33 Yang C, Huang B, Bai S, et al. A generalized surface chalcogenation strategy for boosting the electrochemical $\mathrm{N}_{2}$ fixation of metal nanocrystals. Adv Mater, 2020, 32: 2001267

34 Huang Y, Zhu Y, Chen S, et al. Schottky junctions with Bi cocatalyst for taming aqueous phase $\mathrm{N}_{2}$ reduction toward enhanced solar ammonia production. Adv Sci, 2021, 8: 2003626

35 Chen $\mathrm{P}$, Zhang $\mathrm{N}$, Wang $\mathrm{S}$, et al. Interfacial engineering of cobalt sulfide/graphene hybrids for highly efficient ammonia electrosynthesis. Proc Natl Acad Sci USA, 2019, 116: 6635-6640

36 Li X, Xiao L, Zhou L, et al. Adaptive bifunctional electrocatalyst of amorphous CoFe oxide @2D black phosphorus for overall water splitting. Angew Chem Int Ed, 2020, 59: 21106-21113

37 Deng S, Yuan Z, Tie Z, et al. Electrochemically induced metal-organicframework-derived amorphous $\mathrm{V}_{2} \mathrm{O}_{5}$ for superior rate aqueous zinc-ion batteries. Angew Chem Int Ed, 2020, 59: 22002-22006 
38 Hou Y, Huang YB, Liang YL, et al. Unraveling the reactivity and selectivity of atomically isolated metal-nitrogen sites anchored on porphyrinic triazine frameworks for electroreduction of $\mathrm{CO}_{2}$. CCS Chem, 2019, 1: 384-395

39 Pi Y, Guo J, Shao Q, et al. All-inorganic $\mathrm{SrSnO}_{3}$ perovskite nanowires for efficient $\mathrm{CO}_{2}$ electroreduction. Nano Energy, 2019, 62: 861-868

40 Chen G, Zhou W, Guan D, et al. Two orders of magnitude enhancement in oxygen evolution reactivity on amorphous $\mathrm{Ba}_{0.5} \mathrm{Sr}_{0.5} \mathrm{Co}_{0.8} \mathrm{Fe}_{0.2} \mathrm{O}_{3-\delta}$ nanofilms with tunable oxidation state. Sci Adv, 2017, 3: e1603206

41 Zhang J, Shang X, Ren $\mathrm{H}$, et al. Modulation of inverse spinel $\mathrm{Fe}_{3} \mathrm{O}_{4}$ by phosphorus doping as an industrially promising electrocatalyst for hydrogen evolution. Adv Mater, 2019, 31: 1905107

42 Huang LB, Zhao L, Zhang Y, et al. Self-Limited on-site conversion of $\mathrm{MoO}_{3}$ nanodots into vertically aligned ultrasmall monolayer $\mathrm{MoS}_{2}$ for efficient hydrogen evolution. Adv Energy Mater, 2018, 8: 1800734

43 Han HS, Choi H, Mhin S, et al. Advantageous crystalline-amorphous phase boundary for enhanced electrochemical water oxidation. Energy Environ Sci, 2019, 12: 2443-2454

44 Jenkinson KJ, Wagner A, Kornienko N, et al. A one-pot route to faceted $\mathrm{FePt}-\mathrm{Fe}_{3} \mathrm{O}_{4}$ dumbbells: Probing morphology-catalytic activity effects in $\mathrm{O}_{2}$ reduction catalysis. Adv Funct Mater, 2020, 30: 2002633

45 Zhang J, Ji Y, Wang $\mathrm{P}$, et al. Adsorbing and activating $\mathrm{N}_{2}$ on heterogeneous $\mathrm{Au}-\mathrm{Fe}_{3} \mathrm{O}_{4}$ nanoparticles for $\mathrm{N}_{2}$ fixation. Adv Funct Mater, 2020, 30: 1906579

46 Jiao S, Yao Z, Li M, et al. Accelerating oxygen evolution electrocatalysis of two-dimensional $\mathrm{NiFe}$ layered double hydroxide nanosheets via space-confined amorphization. Nanoscale, 2019, 11: 18894-18899

47 Zhang $\mathrm{H}, \mathrm{Wu} \mathrm{W}$, Liu $\mathrm{Q}$, et al. Interlayer engineering of $\mathrm{a}-\mathrm{MoO}_{3}$ modulates selective hydronium intercalation in neutral aqueous electrolyte. Angew Chem Int Ed, 2021, 60: 896-903

48 Zhang L, Ji X, Ren X, et al. Electrochemical ammonia synthesis via nitrogen reduction reaction on a $\mathrm{MoS}_{2}$ catalyst: Theoretical and experimental studies. Adv Mater, 2018, 30: 1800191

Acknowledgements This work was supported by the National Natural Science Foundation of China (U2032149), Shenzhen Science and Technology Project (JCYJ20180507182246321), Hunan Provincial Natural Science Foundation of China (2020JJ2001), Hefei National Laboratory for Physical Sciences at the Microscale (KF2020108), the Fundamental Research Funds for the Central Universities, and China Postdoctoral Science Foundation (2019M663058 and 2019M652749).

Author contributions $\quad \mathrm{Fu} \mathrm{X}$ and Jiao $\mathrm{S}$ performed the experiments. Jiao $\mathrm{S}$ wrote the manuscript. Zeng $\mathrm{YJ}$ and Huang $\mathrm{H}$ supervised the project. All authors contributed to the general discussion.

Conflict of interest The authors declare that they have no conflict of interest.

Supplementary information Supporting data are available in the online version of the paper.

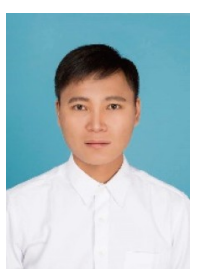

Shilong Jiao received his $\mathrm{PhD}$ degree in materials science from Shandong University. He is currently a postdoc fellow at Shenzhen University. His current research interests include the preparation and characterization of nanocatalysts with various defects and the relationship between structural defects and the electrocatalytic performance of renewable energy-related reactions.

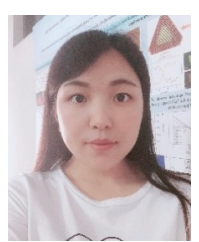

Xianwei Fu received her BS degree from Shanxi Datong University, Datong, China, in 2013, and PhD degree from Shandong University, Jinan, China, in 2018. Now, she is a full postdoctoral fellow at Henan University. Her research interests focus on the fabrication and characterization of low-dimensional material-based optoelectronic devices.

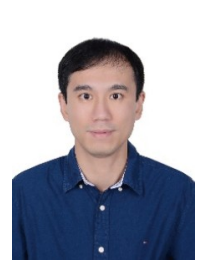

Yu-Jia Zeng is a professor at Shenzhen University. He received his bachelor's degree in materials science and engineering and his $\mathrm{PhD}$ degree in materials physics and chemistry both from Zhejiang University. After graduation, he worked at the Department of Physics of KU Leuven as a postdoctoral fellow and a research associate. His research interests include low-dimensional materials, semiconductors in particular, for optoelectronics and spintronics.

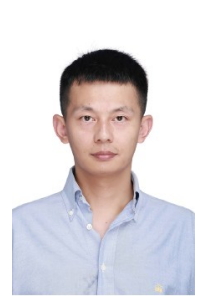

Hongwen Huang is now a full professor at Hunan University. He received his bachelor's degree in materials science and engineering from the South China University of Technology in 2009 and his $\mathrm{PhD}$ degree in materials science and engineering from Zhejiang University in 2015. From 2012 to 2014, he studied at Georgia Institute of Technology under the supervision of Prof. Younan Xia. After graduation, he worked at the University of Science and Technology of China as a postdoctoral fellow from 2015 to 2017 and joined Hunan University in 2017. His research interests include the controlled growth of nanocrystals and their applications in energy-related electrocatalysis.

\section{非周期性原子排列实现电化学氮还原和析氧反应性 能的增强}

焦世龙 ${ }^{1,2,3 \dagger}$, 付现伟 ${ }^{4 \dagger}$, 阮双琛 ${ }^{1}$, 曾昱嘉 ${ }^{*}$, 黄宏文 ${ }^{2,5^{*}}$

摘要 制备低成本的高效电催化剂是缓解目前面临的环境能源危机的 重要方式. 通过无定型化和构建异质结构可以有效地改变催化剂的电 子结构, 从而实现电催化性能的稳步提升. 但是对于两者之间协同作用 的研究则相对较少. 在本文中, 我们构建了富含异质结构的无定型 FeMo基(a-FeMo)电催化剂, 并系统评估了该催化剂的氮气还原和析氧 反应性能. 得益于无定型结构引起的活性位点数目的增多和异质结构 引发的电子的重新分布, a-FeMo催化剂表现出优异的电化学催化性能. 在-0.1 V vs. RHE的电势下, a-FeMo催化剂表现出 $29.15 \%$ 的法拉第效率 和71.78 $\mu_{\mathrm{NH}_{3}} \mathrm{mg}_{\mathrm{cat}}{ }^{-1} \mathrm{~h}^{-1}$ 的氨气产率. 这为合理设计具有低成本优势、 高活性和长时间稳定性的优异催化剂提供了有益的参考. 